\title{
T2-mapping in volunteers: influence of sequence, spatial orientation and interindividual variability
}

\author{
Ralf Wassmuth*, Jeanette Schulz-Menger \\ From 2011 SCMR/Euro CMR Joint Scientific Sessions \\ Nice, France. 3-6 February 2011
}

\section{Background}

CMR T2-mapping is a promising tool for characterizing myocardial edema. We applied T2-mapping in volunteers to compare two mapping sequences and to assess feasibility, reproducibility and spatial homogeneity.

\section{Methods}

We scanned 26 volunteers (10 female, 20-70 years, mean $32 \pm 13$ years, median 28 years, BMI $23 \pm 3 \mathrm{~kg} / \mathrm{m} 2$ ) with a normal ECG, no cardiac disease and no symptoms of inflammation.

Using a $1.5 \mathrm{~T}$ scanner and a dedicated 12-element cardiac coil we applied a FLASH-based and SSFP-based mapping sequence in midventricular short axis (SAX) and four-chamber-view (4CV). The map was based on three images with an echo time of 0, 24 and $55 \mathrm{~ms}$. Spatial resolution was $2.1 \mathrm{~mm} /$ pixel. Scan time was 12 heart beats.

In Osirix 3.3.2. we manually drew 6 segments in SAX and $4 \mathrm{CV}$, each. Additionally, we drew a global region of interest (ROI) covering the whole LV myocardium in SAX and $4 \mathrm{CV}$. The same investigator analyzed 10 data sets twice. 5 volunteers were scanned twice on separate days. Results were compared with a paired student t-test.

\section{Results}

After excluding one subject due to obvious pathologies, 25 datasets with sufficient image quality were evaluated.

Table 1 gives mean and range for global ROI.

FLASH and SSFP correlated better in $4 \mathrm{CV}$ than in SAX (correlation coefficient 0.92 vs. 0.80; $\mathrm{p}<0.04$ ). $\mathrm{T} 2$-values did not correlate with heart rate $(\mathrm{p}=0.3)$.

With both sequences anteroseptal segments had higher T2-values than inferior and inferolateral

Table 1

\begin{tabular}{llll}
\hline & FLASH & SSFP & \\
\hline SAX & $52 \pm 8 ; 42-60$ & $57 \pm 10 ; 46-69$ & $p<0.001$ \\
$4 C V$ & $58 \pm 10 ; 47-74$ & $61 \pm 14 ; 51-80$ & $p<0.005$ \\
& $p<0.001$ & $p<0.001$ & \\
\hline
\end{tabular}

segments in SAX (for FLASH $58 \pm 6$ vs. 48 $\pm 4 ; \mathrm{p}<0.001$ ). In $4 \mathrm{CV}$ the basal septum had higher T2-values than the anterolateral segment with FLASH ( $62 \pm 7$ vs. $54 \pm 8 \mathrm{~ms}$; $\mathrm{p}<0.001$ ), but not with SSFP ( $58 \pm 6$ vs. $60 \pm 11 \mathrm{~ms} ; \mathrm{p}=0.3$ ). Mean absolute difference between a single segment and a global measurement was $4 \pm 1 \mathrm{~ms}$ and $3 \pm 1 \mathrm{~ms}$ for FLASH and SSFP in SAX and $5 \pm 2 \mathrm{~ms}$ for FLASH and SSFP in 4 CV $(\mathrm{p}=0.3)$.

Mean difference for repeated analysis was $1.6 \pm 1.9 \mathrm{~ms}$ (correlation coefficient 0.9 ) and $2.2 \pm 2.2 \mathrm{~ms}$ (correlation coefficient 0.7 ) for repeated scans.

\section{Conclusion}

T2-mapping is feasible with low intraobserver variability and does not depend on heart rate. SSFP-based T2mapping resulted in slightly higher values than FLASH. Mapping in $4 \mathrm{CV}$ resulted in higher T2-values than in SAX. We could detect small spatial differences across the heart. However, these intraindividual spatial variations were smaller than considerable interindividual variability.

Published: 2 February 2011

doi:10.1186/1532-429X-13-S1-P61

Cite this article as: Wassmuth and Schulz-Menger: T2-mapping in

volunteers: influence of sequence, spatial orientation and

interindividual variability. Journal of Cardiovascular Magnetic Resonance 2011 13(Suppl 1):P61.

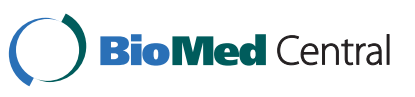

